\title{
A New Optimal Family of Schröder's Method for Multiple Zeros
}

\author{
Ramandeep Behl ${ }^{1, *}$, Arwa Jeza Alsolami ${ }^{1}$, Bruno Antonio Pansera ${ }^{2}{ }^{\complement}$, Waleed M. Al-Hamdan ${ }^{1}$, \\ Mehdi Salimi ${ }^{2,3}$ and Massimiliano Ferrara ${ }^{2,4}$ (iD) \\ 1 Department of Mathematics, Faculty of Science, King Abdulaziz University, P.O. Box 80203, \\ Jeddah 21589, Saudi Arabia; ramanbehl87@gmail.com (A.J.A.); rlal@kau.edu.sa (W.M.A.-H.) \\ 2 Department of Law, Economics and Human Sciences \& Decisions Lab, University Mediterranea of Reggio \\ Calabria, 89125 Reggio Calabria, Italy; bruno.pansera@unirc.it (B.A.P.); mehdi.salimi@unirc.it (M.S.); \\ massimiliano.ferrara@unirc.it (M.F.) \\ 3 Center for Dynamics and Institute for Analysis, Department of Mathematics, Technische Universität \\ Dresden, 01062 Dresden, Germany \\ 4 ICRIOS-The Invernizzi Centre for Research on Innovation, Organization, Strategy and Entrepreneurship, \\ Department of Management and Technology, Bocconi University, Via Sarfatti, 25, 20136 Milano, Italy \\ * Correspondence: ramanbehl87@yahoo.in
}

Received: 25 August 2019; Accepted: 24 October 2019; Published: 8 November 2019

\begin{abstract}
Here, we suggest a high-order optimal variant/modification of Schröder's method for obtaining the multiple zeros of nonlinear uni-variate functions. Based on quadratically convergent Schröder's method, we derive the new family of fourth -order multi-point methods having optimal convergence order. Additionally, we discuss the theoretical convergence order and the properties of the new scheme. The main finding of the present work is that one can develop several new and some classical existing methods by adjusting one of the parameters. Numerical results are given to illustrate the execution of our multi-point methods. We observed that our schemes are equally competent to other existing methods.
\end{abstract}

Keywords: efficiency index; nonlinear uni-variate functions; Schröder's method; optimal order of convergence

\section{Introduction}

There are several issues from chemistry, physics, applied mathematics, scientific computing, economics and engineering that can be transformed to

$$
\Theta(x)=0 .
$$

It is almost inaccessible to find the solution by an analytical approach. Therefore, we worry about the iterative schemes to find the multiple solution $r_{m}$ with known multiplicity $m>1$ of the uni-variate function in Equation (1).

Construction of the iterative schemes [1-3] (that do not essentially require the value of second-order derivative of $\Theta$ ) having optimal convergence order [4] is the foremost and most difficult issue in the region of numerical analysis. It is also interesting with practical perspective because sometimes it is very difficult to compute the second-order derivative.

Some high-order variants and modifications of Newton's method that can solve multiple roots are suggested and investigated [5-11]. Out of them, only two iterative methods by Li et al. [6,7], Sharma and Sharma [8], and Zhou et al. [9] have optimal fourth-order convergence. We have very limited fourth- or high-order schemes until now that are capable of solving multiple roots. 
Khattri and Abbasbandy [12] developed the following scheme for obtaining optimal families of fourth-order methods for simple zeros of uni-variate functions

$$
\left\{\begin{aligned}
y_{l} & =x_{l}-\frac{2}{3} \frac{\Theta\left(x_{l}\right)}{\Theta^{\prime}\left(x_{l}\right)} \\
x_{l+1} & =x_{l}-\frac{\Theta\left(x_{l}\right)}{\Theta^{\prime}\left(x_{l}\right)}\left[1+\sum_{j=1}^{4} \alpha_{i}\left(\frac{\Theta^{\prime}\left(y_{l}\right)}{\Theta^{\prime}\left(x_{l}\right)}\right)^{j}\right],
\end{aligned}\right.
$$

where $\alpha_{j} \in \mathbb{R}$.

Zhou et al. [9] presented an optimal scheme of fourth-order that needs one value of $\Theta$ and two of derivative $\Theta^{\prime}$ at each step and is defined as:

$$
\left\{\begin{array}{c}
y_{l}=x_{l}-\frac{2 m}{m+2} \frac{\Theta\left(x_{l}\right)}{\Theta^{\prime}\left(x_{l}\right)^{\prime}} \\
x_{l+1}=x_{l}-\frac{\Theta\left(x_{l}\right)}{\Theta^{\prime}\left(x_{l}\right)} Q\left(\frac{\Theta^{\prime}\left(y_{l}\right)}{\Theta^{\prime}\left(x_{l}\right)}\right),
\end{array}\right.
$$

where $m$ denotes the multiplicity of the zero.

No doubt, the above-mentioned scheme given by Zhou et al. [9] is a more general form of the fourth-order iterative schemes. This scheme increases the order of convergence of methods from linear to quartic and gives an optimal family of fourth-order methods. However, the proposed family of methods is dependent upon the weight function. Thus, whenever someone wants to develop a new method from this family, he/she has to construct a new weight function that employs the hypotheses mentioned in [9]. It is not easily accessible to obtain such a weight function. Therefore, this is the main drawback of this scheme.

We are keen to construct a new general class of Schröder's method where there is no need for the weight function(s) to develop new methods having optimal fourth-order convergence. For this, we extend the idea of Khattri and Abbasbandy [12] for the multiple zeros. The principle benefit of the scheme is that we have two free disposable parameters. Therefore, one can obtain several new and some existing optimal families by adjusting one of the parameters, unlike the weight function(s) in [9]. Our iterative techniques are equally competent to the existing schemes.

\section{Development of the Fourth-Order Methods}

Here, we suggest a new optimal class of Schröder's method. For this, we consider a Schröder's method [13] that is defined as follows:

$$
x_{l+1}=x_{l}-\frac{\Theta\left(x_{l}\right) \Theta^{\prime}\left(x_{l}\right)}{\Theta^{\prime 2}\left(x_{l}\right)-\Theta\left(x_{l}\right) \Theta^{\prime \prime}\left(x_{l}\right)} .
$$

The principle advantages of this method are that it does not depend upon the multiplicity of the required zero along and it has second-order convergence. The simplest way to obtain this method is to adopt the classical Newton's method to the function $\eta(x)=\frac{\Theta(x)}{\Theta^{\prime}(x)}$ that has a simple zero corresponding to the multiple root of function $\Theta$.

We assume the following Newton-like method for multiple roots

$$
y_{l}=x_{l}-\frac{2 m}{m+2} \frac{\Theta\left(x_{l}\right)}{\Theta^{\prime}\left(x_{l}\right)} .
$$

To abolish second-order derivative from the scheme in Equation (4), expand the function $\Theta^{\prime}\left(y_{l}\right) \equiv$ $\Theta^{\prime}\left(x_{l}-\frac{2 m}{m+2} \frac{\Theta\left(x_{l}\right)}{\Theta^{\prime}\left(x_{l}\right)}\right)$ about the point $x=x_{l}$ by Taylor's series expansion, which leads us to 


$$
\Theta^{\prime}\left(x_{l}-\frac{2 m}{m+2} \frac{\Theta\left(x_{l}\right)}{\Theta^{\prime}\left(x_{l}\right)}\right) \approx \Theta^{\prime}\left(x_{l}\right)-\frac{2 m}{m+2} \frac{\Theta\left(x_{l}\right) \Theta^{\prime \prime}\left(x_{l}\right)}{\Theta^{\prime}\left(x_{l}\right)},
$$

further yielding

$$
\Theta^{\prime \prime}\left(x_{l}\right) \approx \frac{(m+2) \Theta^{\prime}\left(x_{l}\right)\left[\Theta^{\prime}\left(x_{l}\right)-\Theta^{\prime}\left(y_{l}\right)\right]}{2 m \Theta\left(x_{l}\right)} .
$$

Using Equation (7) in the Schröder's method in Equation (4), we obtain

$$
x_{l+1}=x_{l}-\frac{2 m \Theta\left(x_{l}\right)}{(m-2) \Theta^{\prime}\left(x_{l}\right)+(m+2) \Theta^{\prime}\left(y_{l}\right)} .
$$

The method in Equation (8) has the following error equation

$$
e_{l+1}=\left(\frac{m^{m}(m+2)^{2}+m(m-4)(m+2)^{m}}{m^{m}(m+2)^{2}+m(m-2)(m+2)^{m}}\right) e_{l}+O\left(e_{l}^{2}\right) .
$$

Due to Kung-Traub conjecture [4], Equation (8) is a non optimal scheme because of linear convergence order. Additionally, it uses three functional evaluations at each step. To increase the convergence order of Equation (8), we consider the following iterative scheme

$$
\left\{\begin{aligned}
y_{l} & =x_{l}-\frac{2 m}{m+2} \frac{\Theta\left(x_{l}\right)}{\Theta^{\prime}\left(x_{l}\right)}, \\
x_{l+1} & =x_{l}-\frac{2 m \gamma \Theta\left(x_{l}\right)}{(m-2) \beta \Theta^{\prime}\left(x_{l}\right)+(m+2) \Theta^{\prime}\left(y_{l}\right)} \sum_{i=0}^{2} \alpha_{i}\left(\frac{\Theta^{\prime}\left(y_{l}\right)}{\Theta^{\prime}\left(x_{l}\right)}\right)^{i},
\end{aligned}\right.
$$

where $\gamma, \beta, \alpha_{0}, \alpha_{1}, \alpha_{2} \in \mathbb{R}$ are free variables. These variables are responsible for obtaining optimal fourth-order convergence. Theorem 1 illustrates the restrictions on the parameters, so the above scheme in Equation (9) reaches at optimal convergence order.

Theorem 1. Let $\Theta: \mathbb{A} \subseteq \mathbb{C} \rightarrow \mathbb{C}$ be a holomorphic function in the region $\mathbb{A}$ that encloses the desired multiple zero of $\Theta(x)$, (say $x=r_{m}$ ) with known multiplicity $m \geq 1$. Then, the family in Equation (9) has fourth-order of convergence, when

$$
\left\{\begin{array}{c}
\gamma=-\frac{m\left(m^{2}-4\right)}{4\left(m \alpha_{1}+2 u(m+3) \alpha_{2}\right)}, \alpha_{1} \neq-\frac{2 u(m+3) \alpha_{2}}{m}, \\
\beta=-\frac{u(m+2)\left[m^{3} \alpha_{1}+2 u\left(m^{3}+3 m^{2}+2 m-4\right) \alpha_{2}\right]}{m^{2}(m-2)\left(m \alpha_{1}+2 u(m+3) \alpha_{2}\right)} \\
\alpha_{0}=-\frac{u\left(m^{4} \alpha_{1}+u\left(m^{4}+2 m^{3}+4 m^{2}+8 m+16\right) \alpha_{2}\right)}{m^{3}(m-2)}
\end{array}\right.
$$

where $u=\left(\frac{m}{m+2}\right)^{m}$ and $\alpha_{1}, \alpha_{2} \in \mathbb{R}$ are free variables.

Proof. We assume that $x=r_{m}$ is a multiple zero of multiplicity $m$ of $\Theta(x)$. Now, expand $\Theta\left(x_{l}\right)$ and $\Theta^{\prime}\left(x_{l}\right)$ about a point $x=r_{m}$ with the help of the Taylor's series expansion. Then, we yield

$$
\Theta\left(x_{l}\right)=\frac{\Theta^{(m)}\left(r_{m}\right)}{m !} e_{l}^{m}\left(1+c_{1} e_{l}+c_{2} e_{l}^{2}+c_{3} e_{l}^{3}+c_{4} e_{l}^{4}+O\left(e_{l}^{5}\right)\right)
$$

and

$$
\Theta^{\prime}\left(x_{l}\right)=\frac{\Theta^{(m-1)}\left(r_{m}\right)}{(m-1) !} e_{l}^{m-1}\left(1+\frac{m+1}{m} c_{1} e_{l}+\frac{m+2}{m} c_{2} e_{l}^{2}+\frac{m+3}{m} c_{3} e_{l}^{3}+\frac{m+4}{m} c_{4} e_{l}^{4}+O\left(e_{l}^{5}\right)\right),
$$


respectively, where $c_{k}=\frac{m !}{(m+k) !} \frac{\Theta^{(m+k)}\left(r_{m}\right)}{\Theta^{(m)}\left(r_{m}\right)}, k=1,2,3,4, \ldots$ are the asymptotic error constants.

By adopting Equations (11) and (12), we obtain

$$
\frac{\Theta\left(x_{l}\right)}{\Theta^{\prime}\left(x_{l}\right)}=\frac{1}{m} e_{l}-\frac{c_{1}}{m^{2}} e_{l}^{2}+\left(\frac{(m+1) c_{1}^{2}-2 m c_{2}}{m^{3}}\right) e_{l}^{3}+O\left(e_{l}^{4}\right) .
$$

Furthermore, we have

$$
\begin{aligned}
\frac{2 m \alpha \Theta\left(x_{l}\right)}{(m-2) \beta \Theta^{\prime}\left(x_{l}\right)+(m+2) \Theta^{\prime}\left(y_{l}\right)} & =\left(\frac{2 m \alpha}{m(m-2) \beta+u(m+2)^{2}}\right) e_{l} \\
& -\left(\frac{2\left(m^{2}(m-2) \beta+u\left(m^{3}+4 m^{2}-8\right)\right) \alpha c_{1}}{m\left(m(m-2) \beta+u(m+2)^{2}\right)^{2}}\right) e_{l}^{2}+O\left(e_{l}^{3}\right),
\end{aligned}
$$

and

$$
\begin{aligned}
\frac{\Theta^{\prime}\left(y_{l}\right)}{\Theta^{\prime}\left(x_{l}\right)}= & \left(\frac{m}{m+2}\right)^{m-1}-\frac{4 u c_{1}}{m^{3}} e_{l}+4 u\left(\frac{(m+2) c_{1}^{2}-2 m^{2} c_{2}}{m^{5}}\right) e_{l}^{2}-\frac{8 u}{3 m^{7}(m+2)^{2}}\left[( m + 2 ) ^ { 2 } \left(m^{4}-m^{3}\right.\right. \\
& \left.\left.+5 m^{2}+m+6\right) c_{1}^{3}-3 m^{2}(m+2)^{2}\left(m^{2}+4\right) c_{1} c_{2}+3 m^{4}\left(m^{2}+6 m+6\right) c_{3}\right] e_{l}^{3}+O\left(e_{l}^{4}\right) .
\end{aligned}
$$

Using Equations (14) and (15) in the scheme in Equation (9), we have

$$
\begin{aligned}
e_{l+1}= & e_{l}-\frac{2 m \alpha \Theta\left(x_{l}\right)}{(m-2) \beta \Theta^{\prime}\left(x_{l}\right)+(m+2) \Theta^{\prime}\left(y_{l}\right)} \sum_{i=0}^{2} \alpha_{i}\left(\frac{\Theta^{\prime}\left(y_{l}\right)}{\Theta^{\prime}\left(x_{l}\right)}\right)^{i}, \\
= & \left(1-\frac{2 m \alpha\left(\alpha_{0}+\left(\frac{m}{m+2}\right)^{m-1} \alpha_{1}+\left(\frac{m}{m+2}\right)^{2(m-1)} \alpha_{2}\right)}{m(m-2) \beta+(m+2)^{2} u}\right) e_{l}+\left(\frac{8 u \alpha\left(m \alpha_{1}+2 u(m+2) \alpha_{2}\right)}{m^{3}\left(m(m-2) \beta+(m+2)^{2} u\right)}\right. \\
& \left.+\frac{2\left(\alpha_{0}+\left(\frac{m}{m+2}\right)^{m-1} \alpha_{1}+\left(\frac{m}{m+2}\right)^{2(m-1)} \alpha_{2}\right)\left(m^{2}(m-2) \beta+u\left(m^{3}+4 m^{2}-8\right)\right)}{m\left(m(m-2) \beta+(m+2)^{2} u\right)^{2}}\right) c_{1} e_{l}^{2} \\
+ & \left(k_{1} c_{2}-k_{2} c_{1}^{2}\right) e_{l}^{3}+O\left(e_{l}^{4}\right),
\end{aligned}
$$

where

$$
\begin{aligned}
k_{1}= & \frac{1}{m^{3}\left(m(m-2) \beta+u(m+2)^{2}\right)^{2}}\left[4 \left(m^{4}(m-2) \beta \alpha_{0}+(m+2)^{3}\left(m^{2}+2 m+4\right) u^{3} \alpha_{2}\right.\right. \\
& \left.\left.+m(m+2) u^{2}\left(m(m+2)^{2} \alpha_{1}\left(m^{3}+4 m-16\right) \beta \alpha_{2}\right)+m^{2} u\left(\left(m^{3}-8\right) \beta \alpha_{1}+\left(m^{3}+4 m^{2}-8\right) \alpha_{0}\right)\right)\right] \alpha, \\
k_{2}= & \frac{2}{m^{5}\left(m(m-2) \beta+u(m+2)^{2}\right)^{3}}\left[( m + 2 ) ^ { 2 } u ^ { 2 } \left(m^{3}\left(m^{4}+5 m^{3}+4 m^{2}-8 m-16\right)+(m+2)^{3} u\left(m^{3}(m+1) \alpha_{1}\right)\right.\right. \\
& \left.+u\left(m^{4}+3 m^{3}+6 m^{2}+4 m+8\right)\right)+2 m u \beta\left(m^{2}-4\right)\left(m^{2}\left(m^{4}+3 m^{3}-2 m-4\right) \alpha_{0}\right. \\
& \left.+u\left(u(m+2)^{2}\left(m^{4}+3 m^{3}+8 m^{2}+6 m+12\right) \alpha_{2}+m^{2}\left(m^{4}+5 m^{3}+10 m^{2}+10 m+8\right) \alpha_{1}\right)\right) \\
& +m^{2}(m-2)^{2} \beta^{2}\left(m^{4}(m+1) \alpha_{0}+u\left(m\left(m^{4}+3 m^{3}+6 m^{2}+4 m+8\right) \alpha_{1}\right.\right. \\
& \left.\left.\left.+u\left(m^{5}+5 m^{4}+16 m^{3}+28 m^{2}+32 m+48\right) \alpha_{2}\right)\right)\right] .
\end{aligned}
$$

For attaining fourth-order convergence of the scheme in Equation (9), the coefficients of $e_{l}, e_{l}^{2}$, and $e_{l}^{3}$ should be zero simultaneously. From Equation (16), we obtain the following values of $\alpha, \beta, \alpha_{0}$ involving two free disposable parameters $\alpha_{1}$ and $\alpha_{2}$ 


$$
\left\{\begin{array}{c}
\gamma=-\frac{m\left(m^{2}-4\right)}{4\left(m \alpha_{1}+2 u(m+3) \alpha_{2}\right)}, \alpha_{1} \neq-\frac{2 u(m+3) \alpha_{2}}{m}, \\
\beta=-\frac{u(m+2)\left[m^{3} \alpha_{1}+2 u\left(m^{3}+3 m^{2}+2 m-4\right) \alpha_{2}\right]}{m^{2}(m-2)\left(m \alpha_{1}+2 u(m+3) \alpha_{2}\right)} \\
\alpha_{0}=-\frac{u\left(m^{4} \alpha_{1}+u\left(m^{4}+2 m^{3}+4 m^{2}+8 m+16\right) \alpha_{2}\right)}{m^{3}(m-2)}
\end{array}\right.
$$

where $u=\left(\frac{m}{m+2}\right)^{m}$.

By inserting Equation (17) into Equation (16), we yield

$$
e_{l+1}=\left(\frac{\left.\kappa(m+2)^{2} c_{1}^{3}+3 m^{4}\left(m^{2} \alpha_{1}+2 u\left(m^{2}+2 m+2\right)\right) \alpha_{2}\right)\left(m^{2} c_{3}-(m+2)^{2} c_{1} c_{2}\right)}{3 m^{5}(m+2)^{2}\left(m^{2} \alpha_{1}+2 u\left(m^{2}+2 m+2\right) \alpha_{2}\right)}\right) e_{l}^{4}+O\left(e_{l}^{5}\right),
$$

where $\kappa=m^{3}\left(m^{3}+2 m^{2}+2 m-2\right) \alpha_{1}+2 u\left(m^{6}+4 m^{5}+8 m^{4}+6 m^{3}-16 m+24\right) \alpha_{2}$.

This confirms that the new scheme in Equation (14) attains the optimal convergence order by just consuming three functional evaluations at each step. Hence, it completes the proof.

\section{Particular Forms}

Since we have two free disposable parameters, namely $\alpha_{1}$ and $\alpha_{2}$, we can simply develop several new and some existing classical iterative functions by adjusting one of the free parameters in the scheme in Equation (9).

\section{Special cases:}

Case 1. For $\alpha_{2}=1$, general class (9) gives

$$
\left\{\begin{aligned}
y_{l} & =x_{l}-\frac{2 m}{m+2} \frac{\Theta\left(x_{l}\right)}{\Theta^{\prime}\left(x_{l}\right)^{\prime}} \\
x_{l+1} & =x_{l}+\frac{m \Theta\left(x_{l}\right)}{2 \Theta^{\prime}\left(x_{l}\right)^{2}}\left[\frac{\Theta^{\prime}\left(x_{l}\right)^{2}\left(m^{4}+2 m^{3}+4 m^{2}+8 m+16\right) u^{2}+\alpha_{1} \Theta^{\prime}\left(x_{l}\right) m^{3}\left(\Theta^{\prime}\left(x_{l}\right) m u-\Theta^{\prime}\left(y_{l}\right)(m-2)\right)-\Theta^{\prime}\left(y_{l}\right)^{2}(m-2) m^{3}}{2 u\left(\Theta^{\prime}\left(x_{l}\right) u\left(m^{3}+3 m^{2}+2 m-4\right)-\Theta^{\prime}\left(y_{l}\right) m^{2}(m+3)\right)-\alpha_{1} m^{3}\left(\Theta^{\prime}\left(y_{l}\right)-\Theta^{\prime}\left(x_{l}\right) u\right)}\right] .
\end{aligned}\right.
$$

Equation (18) is a new fourth-order optimal family for multiple roots.

\section{Special cases of the scheme in Equation (18):}

(i) For $\alpha_{1}=0$, Equation (18) leads us to

$$
\left\{\begin{aligned}
y_{l} & =x_{l}-\frac{2 m}{m+2} \frac{\Theta\left(x_{l}\right)}{\Theta^{\prime}\left(x_{l}\right)}, \\
x_{l+1} & =x_{l}+\frac{m \Theta\left(x_{l}\right)}{4 u \Theta^{\prime}\left(x_{l}\right)^{2}}\left[\frac{u^{2} \Theta^{\prime}\left(x_{l}\right)^{2}\left(m^{4}+2 m^{3}+4 m^{2}+8 m+16\right)-\Theta^{\prime}\left(y_{l}\right)^{2}(m-2) m^{3}}{\Theta^{\prime}\left(x_{l}\right) u\left(m^{3}+3 m^{2}+2 m-4\right)-\Theta^{\prime}\left(y_{l}\right) m^{2}(m+3)}\right],
\end{aligned}\right.
$$

which is another new fourth-order optimal technique.

(ii) For $\alpha_{1}=-\frac{u\left(m^{4}+2 m^{3}+4 m^{2}+8 m+16\right)}{m^{4}}$, the family in Equation (18) provides the following new fourth-order optimal scheme

$$
\left\{\begin{array}{c}
y_{l}=x_{l}-\frac{2 m}{m+2} \frac{\Theta\left(x_{l}\right)}{\Theta^{\prime}\left(x_{l}\right)}, \\
x_{l+1}=x_{l}+\frac{m \Theta\left(x_{l}\right) \Theta^{\prime}\left(y_{l}\right)}{2 u \Theta^{\prime 2}\left(x_{l}\right)}\left[\frac{\left(m^{4}+2 m^{3}+4 m^{2}+8 m+16\right) u \Theta^{\prime}\left(x_{l}\right)-m^{4} \Theta^{\prime}\left(y_{l}\right)}{(m+2)^{3} u \Theta^{\prime}\left(x_{l}\right)-\left(m^{3}+6 m^{2}+8 m+8\right) \Theta^{\prime}\left(y_{l}\right)}\right] .
\end{array}\right.
$$

(iii) For $\alpha_{1}=-\frac{2 u\left(m^{3}+3 m^{2}+2 m-4\right)}{m^{3}}$, the scheme in Equation (18) further yields 


$$
\left\{\begin{aligned}
y_{l} & =x_{l}-\frac{2 m}{m+2} \frac{\Theta\left(x_{l}\right)}{\Theta^{\prime}\left(x_{l}\right)} \\
x_{l+1} & =x_{l}-\frac{m}{8}\left[m^{3} u^{-1}\left(\frac{\Theta^{\prime}\left(y_{l}\right)}{\Theta^{\prime}\left(x_{l}\right)}\right)^{2}-2\left(m^{3}+3 m^{2}+2 m-4\right) \frac{\Theta^{\prime}\left(y_{l}\right)}{\Theta^{\prime}\left(x_{l}\right)}+u(m+2)^{3}\right] \frac{\Theta^{\prime}\left(x_{l}\right)}{\Theta^{\prime}\left(y_{l}\right)} .
\end{aligned}\right.
$$

This is a well-known fourth-order optimal technique developed by Zhou et al. [9].

Case 2. For $\alpha_{1}=1$, the general class in Equation (9) leads us to another new optimal fourth-order scheme:

$$
\left\{\begin{array}{l}
y_{l}=x_{l}-\frac{2 m}{m+2} \frac{\Theta\left(x_{l}\right)}{\Theta^{\prime}\left(x_{l}\right)^{\prime}} \\
x_{l+1}=x_{l}+\frac{m \Theta\left(x_{l}\right)}{2 \Theta^{\prime 2}\left(x_{l}\right)}\left[\frac{\alpha_{2}\left(u^{2} \Theta^{\prime}\left(x_{l}\right)^{2}\left(m^{4}+2 m^{3}+4 m^{2}+8 m+16\right)-\Theta^{\prime}\left(y_{l}\right)^{2}(m-2) m^{3}\right)+\Theta^{\prime}\left(x_{l}\right) m^{3}\left(\Theta^{\prime}\left(x_{l}\right) m u-\Theta^{\prime}\left(y_{l}\right)(m-2)\right)}{2 \alpha_{2} u\left(\Theta^{\prime}\left(x_{l}\right) u\left(m^{3}+3 m^{2}+2 m-4\right)-\Theta^{\prime}\left(y_{l}\right) m^{2}(m+3)\right)-m^{3}\left(\Theta^{\prime}\left(y_{l}\right)-\Theta^{\prime}\left(x_{l}\right) u\right)}\right] .
\end{array}\right.
$$

\section{Sub special case of the family in Equation (22):}

(i) For $\alpha_{2}=0$, the family in Equation (22) gives a well-known fourth-order method developed by Li et al. [7], which is defined as follows:

$$
\left\{\begin{array}{c}
y_{l}=x_{l}-\frac{2 m}{m+2} \frac{\Theta\left(x_{l}\right)}{\Theta^{\prime}\left(x_{l}\right)} \\
x_{l+1}=x_{l}-\frac{m \Theta\left(x_{l}\right)}{2 \Theta^{\prime}\left(x_{l}\right)}\left[\frac{(m-2) \Theta^{\prime}\left(y_{l}\right)-m u \Theta^{\prime}\left(x_{l}\right)}{u \Theta^{\prime}\left(x_{l}\right)-\Theta^{\prime}\left(y_{l}\right)}\right] .
\end{array}\right.
$$

It is obvious to observe that each step of the schemes in Equations (18)-(20) and (22) consumr only three functional evaluations each iteration, viz. one value of $\Theta(x)$ and two of $\Theta^{\prime}(x)$. For checking the efficiency of the new technique in Equation (9), we adopt the efficiency index $a^{\frac{1}{\rho}}$ (for more details, please see [14]). Thus, we have $a=4$ and $\rho=3$ that provide us $E \cong 1.587$, which is far better than third-order and modified Newton's technique $E \cong 1.442$ and $E \cong 1.414$, respectively.

\section{Numerical Results}

We verified the potency of the new optimal techniques. We computee them with the iterative expressions (35) and (37) described by Sharifi et al. in [15], called by (SH1) and (SH2), respectively. In addition, we considered method (11) from Zhou et al. [9] (defined by ZS) for comparison with our methods. Further, we compared them with expressions (69) and (75) mentioned by Li et al. [6], called by (LS1) and (LS2), respectively. Finally, we computed them with expression (1) depicted by Sharma and Sharma [8], known as (SAS).

In Tables 1-6, we display the values of iterates $\left(x_{l}\right)$, the absolute residual errors $\left|f\left(x_{l}\right)\right|$ and the absolute errors $\left|x_{l+1}-x_{l}\right|$. We adopted the the following formula [16], for the computational order of convergence (COC)

$$
\rho \approx \frac{\ln \left|\left(x_{l+1}-r_{m}\right) /\left(x_{l}-r_{m}\right)\right|}{\ln \left|\left(x_{l}-r_{m}\right) /\left(x_{l-1}-r_{m}\right)\right|} .
$$

In the above COC, we need the exact zero $r_{m}$. However, there are several practical situations where the exact solution is not accessible. Therefore, Ezquerro and Hernández, [17] suggested the following technique

$$
\rho \approx \frac{\ln \left|\check{e}_{l+1} / \check{e}_{l}\right|}{\ln \left|\check{e}_{l} / \check{e}_{l-1}\right|}
$$

where $\breve{e}_{l}=x_{l}-x_{l-1}$ and there is no need of an exact zero.

During the current numerical experiments with programming language Mathematica (Version-9), all computations were done with 300 digits of mantissa, which minimizes round-off errors. 
The configuration of the used computer is given below:

Processor: Intel(R) Core(TM) i7-4790 CPU @ 3.60GHz

Made: HP

RAM: 8:00GB

System type: 64-bit-Operating System, x64-based processor.

In addition, the initial guesses of each $\Theta_{i}$ are mentioned in the corresponding table. Further, $\left(b_{1} \pm b_{2}\right)$ express as $\left(b_{1} \times 10^{ \pm b_{2}}\right)$.

\section{Example 1. Study of multi-factor effect}

The mathematical expression of the trajectory of an electron that moves between two parallel sheets along with air gap is defined as follows:

$$
x(t)=x_{0}+\left(v_{0}+e \frac{E_{0}}{m \omega} \sin \left(\omega t_{0}+\alpha\right)\right)\left(t-t_{0}\right)+e \frac{E_{0}}{m \omega^{2}}(\cos (\omega t+\alpha)+\sin (\omega+\alpha)),
$$

where $m$ and $e$ stand for the mass and charge of an electron at the rest position, respectively. In addition, $v_{0}$ and $x_{0}$ are known as the velocity and position of an electron at the initial timing $t_{0}$, respectively. Finally, $E_{0} \sin (\omega t+\alpha)$ denotes a RF electric field among two sheets. With the particular values of these parameters, Equation (18) leads us to

$$
\Theta_{1}(x)=x+\cos (x)-\frac{\pi}{2}
$$

The above function has a solution $x=1.570796326794896619231322$ of multiplicity 3 and numerical results of Equation (25) are depicted in Table 1.

\begin{tabular}{|c|c|c|c|c|c|}
\hline Methods & $l$ & $x_{l}$ & $\left|\Theta\left(x_{l}\right)\right|$ & $\left|x_{l+1}-x_{l}\right|$ & $\rho$ \\
\hline \multirow{4}{*}{ SH1 } & 0 & 1.5 & $5.9(-5)$ & $7.1(-2)$ & \\
\hline & 1 & 1.570797636838370636439511 & $3.7(-19)$ & $1.3(-6)$ & \\
\hline & 2 & 1.570796326794896619231322 & $3.8(-90)$ & $2.8(-30)$ & 5.000 \\
\hline & 3 & 1.570796326794896619231322 & $4.2(-445)$ & $1.4(-148)$ & 5.000 \\
\hline \multirow{4}{*}{ SH2 } & 0 & 1.5 & $5.9(-5)$ & $1.7(-2)$ & \\
\hline & 1 & 1.570796082657860420584872 & $2.4(-21)$ & $2.4(-7)$ & \\
\hline & 2 & 1.570796326794896619231322 & $7.0(-87)$ & $3.5(-29)$ & 3.999 \\
\hline & 3 & 1.570796326794896619231322 & $5.0(-349)$ & $1.4(-116)$ & 4.000 \\
\hline \multirow{4}{*}{$Z S$} & 0 & 1.5 & $5.9(-5)$ & $1.7(-2)$ & \\
\hline & 1 & 1.570796328947672697948766 & $1.7(-27)$ & $2.2(-9)$ & \\
\hline & 2 & 1.570796326794896619231322 & $2.9(-140)$ & $5.6(-47)$ & 5.000 \\
\hline & 3 & 1.570796326794896619231322 & $4.8(-704)$ & $6.6(-235)$ & 5.000 \\
\hline \multirow{4}{*}{$L S 1$} & 0 & 1.5 & $5.9(-5)$ & $1.7(-2)$ & \\
\hline & 1 & 1.570796328947600675637296 & $1.7(-27)$ & $2.2(-9)$ & \\
\hline & 2 & 1.570796326794896619231322 & $2.9(-140)$ & $5.6(-47)$ & 5.000 \\
\hline & 3 & 1.570796326794896619231322 & $4.8(-704)$ & $6.6(-235)$ & 5.000 \\
\hline \multirow{4}{*}{ LS2 } & 0 & 1.5 & $5.9(-5)$ & $1.7(-2)$ & \\
\hline & 1 & 1.570796328947584672461528 & $1.7(-27)$ & $2.2(-9)$ & \\
\hline & 2 & 1.570796326794896619231322 & $2.9(-140)$ & $5.6(-47)$ & 5.000 \\
\hline & 3 & 1.570796326794896619231322 & $4.8(-704)$ & $6.6(-235)$ & 5.000 \\
\hline \multirow{4}{*}{$S A S$} & 0 & 1.5 & $5.9(-5)$ & $1.7(-2)$ & \\
\hline & 1 & 1.570796328947637485401077 & $1.7(-27)$ & $2.2(-9)$ & \\
\hline & 2 & 1.570796326794896619231322 & $2.9(-140)$ & $5.6(-47)$ & 5.000 \\
\hline & 3 & 1.570796326794896619231322 & $4.8(-704)$ & $6.6(-235)$ & 5.000 \\
\hline \multirow{4}{*}{ (19) } & 0 & 1.5 & $5.9(-5)$ & $1.7(-2)$ & \\
\hline & 1 & 1.570796328947576043144954 & $1.7(-27)$ & $2.2(-9)$ & \\
\hline & 2 & 1.570796326794896619231322 & $2.9(-140)$ & $5.6(-47)$ & 5.000 \\
\hline & 3 & 1.570796326794896619231322 & $4.8(-704)$ & $6.6(-235)$ & 5.000 \\
\hline \multirow{4}{*}{$(20)$} & 0 & 1.5 & $5.9(-5)$ & $1.7(-2)$ & \\
\hline & 1 & 1.570796328947556877383065 & $1.7(-27)$ & $2.2(-9)$ & \\
\hline & 2 & 1.570796326794896619231322 & $2.9(-140)$ & $5.6(-47)$ & 5.000 \\
\hline & 3 & 1.570796326794896619231322 & $4.8(-704)$ & $6.6(-235)$ & 5.000 \\
\hline
\end{tabular}

Table 1. Convergence study of distinct iterative functions on $\Theta_{1}(x)$. 
Example 2. Eigen value problem:

We considered a matrix of order $8 \times 8$, defined as follows:

$$
A=\left[\begin{array}{cccccccc}
-12 & -12 & 36 & -12 & 0 & 0 & 12 & 8 \\
148 & 129 & -397 & 147 & -12 & 6 & -109 & -74 \\
72 & 62 & -186 & 66 & -8 & 4 & -54 & -36 \\
-32 & -24 & 88 & -36 & 0 & 0 & 24 & 16 \\
20 & 13 & -45 & 19 & 8 & 6 & -13 & -10 \\
120 & 98 & -330 & 134 & -8 & 24 & -90 & -60 \\
-132 & -109 & 333 & -115 & 12 & -6 & 105 & 66 \\
0 & 0 & 0 & 0 & 0 & 0 & 0 & 4
\end{array}\right] .
$$

We have the following characteristic equation of the above matrix:

$$
\Theta_{2}(x)=x^{8}-36 x^{7}+240 x^{6}+4032 x^{5}-59136 x^{4}+193536 x^{3}+512000 x^{2}-3981312 x+5898240 .
$$

Equation (26) has a zero at $x=4$ of multiplicity $m=3$. The computational results on Equation (26) are mentioned in Table 2.

\begin{tabular}{|c|c|c|c|c|c|}
\hline Methods & $l$ & $x_{l}$ & $\left|\Theta\left(x_{l}\right)\right|$ & $\left|x_{l+1}-x_{l}\right|$ & $\rho$ \\
\hline \multirow{4}{*}{ SH1 } & 0 & 3.9 & $6.7(+1)$ & $1.0(-1)$ & \\
\hline & 1 & 4.000006803578217217648720 & $2.1(-11)$ & $6.8(-6)$ & \\
\hline & 2 & 3.9999999999999999999999991 & $4.7(-65)$ & $9.0(-24)$ & 4.291 \\
\hline & 3 & 4.000000000000000000000000 & $1.3(-279)$ & $2.7(-95)$ & 4.000 \\
\hline \multirow{4}{*}{ SH2 } & 0 & 3.9 & $6.7(+1)$ & $1.0(-1)$ & \\
\hline & 1 & 3.999998660314174738700630 & $1.6(-$ & $1.3(-6)$ & \\
\hline & 2 & 4.000000000000000000000000 & $6.0(-72)$ & $4.5(-26)$ & 3.996 \\
\hline & 3 & 4.000000000000000000000000 & $1.2(-305)$ & $5.7(-104)$ & 4.000 \\
\hline \multirow{4}{*}{ ZS } & 0 & 3.9 & $6.7(+1)$ & $1.0(-1)$ & \\
\hline & 1 & 3.999999608618246693946236 & $3.9(-15)$ & $3.9(-7)$ & \\
\hline & 2 & 4.000000000000000000000000 & $7.0(-80)$ & $1.0(-28)$ & 3.992 \\
\hline & 3 & 4.000000000000000000000000 & $6.8(-339)$ & $4.7(-115)$ & 4.000 \\
\hline \multirow{4}{*}{ LS1 } & 0 & 3.9 & $6.7(+1)$ & $1.0(-1)$ & \\
\hline & 1 & 3.999999619579681070164954 & $3.6(-15)$ & $3.8(-7)$ & \\
\hline & 2 & 4.000000000000000000000000 & $4.5(-80)$ & $8.8(-29)$ & 3.992 \\
\hline & 3 & 4.000000000000000000000000 & $1.1(-339)$ & $2.6(-115)$ & 4.000 \\
\hline \multirow{4}{*}{ LS2 } & 0 & 3.9 & $6.7(+1)$ & $1.0(-1)$ & \\
\hline & 1 & 3.999999622028540284202773 & $3.5(-15)$ & $3.8(-7)$ & \\
\hline & 2 & 4.000000000000000000000000 & $4.1(-80)$ & $8.6(-29)$ & 3.992 \\
\hline & 3 & 4.000000000000000000000000 & $7.4(-340)$ & $2.2(-115)$ & 4.000 \\
\hline \multirow{4}{*}{ SAS } & 0 & 3.9 & & $1.0(-1)$ & \\
\hline & 1 & 3.999999613965262138066513 & $3.8(-15)$ & $3.9(-7)$ & \\
\hline & 2 & 4.000000000000000000000000 & $5.6(-80)$ & $9.5(-29)$ & 3.992 \\
\hline & 3 & 4.000000000000000000000000 & $2.8(-339)$ & $3.5(-115)$ & 4.000 \\
\hline \multirow{4}{*}{ (19) } & 0 & 3.9 & $6.7(+1)$ & $1.0(-1)$ & \\
\hline & 1 & 3.999999623347893731527904 & $3.5(-15)$ & $3.8(-7)$ & \\
\hline & 2 & 4.000000000000000000000000 & $3.9(-80)$ & $8.4(-29)$ & 3.992 \\
\hline & 3 & 4.000000000000000000000000 & $6.0(-340)$ & $2.1(-115)$ & 4.000 \\
\hline \multirow{4}{*}{ (20) } & 0 & 3.9 & $6.7(+1)$ & $1.0(-1)$ & \\
\hline & 1 & 3.999999626281209905722408 & $3.4(-15)$ & $3.7(-7)$ & \\
\hline & 2 & 4.000000000000000000000000 & $3.5(-80)$ & $8.1(-29)$ & 3.992 \\
\hline & 3 & 4.000000000000000000000000 & $3.6(-340)$ & $1.8(-115)$ & 4.000 \\
\hline
\end{tabular}

Table 2. Convergence study of distinct iterative functions on $\Theta_{2}(x)$. 


\section{Example 3. Chemical engineering problem:}

We next assumed a fourth-order polynomial expression from $[18,19]$. That expression outlines the fraction conversion problem of nitrogen and hydrogen when they are transformed into ammonia (such fractions are known as fractional conversion). Adopting $250 \mathrm{~atm}$ and $500^{\circ} \mathrm{C}$ leads to

$$
\Theta_{3}(z)=z^{4}-7.79075 z^{3}+14.7445 z^{2}+2.511 z-1.674 .
$$

Equation (27) has two real and two complex zeros. However, we chose complex zero $\xi=$ $3.9485424455620457727+0.3161235708970163733 i$ to illustrate the application on complex roots.

Table 3. Convergence study of distinct iterative functions on $\Theta_{3}(x)$.

\begin{tabular}{|c|c|c|c|c|c|}
\hline Methods & $l$ & $x_{l}$ & $\left|\Theta\left(x_{l}\right)\right|$ & $\left|x_{l+1}-x_{l}\right|$ & $\rho$ \\
\hline \multirow{4}{*}{ SH1 } & 0 & $3.9+0.25 i$ & $7.3(-1)$ & $8.2(-2)$ & \\
\hline & 1 & $3.948208607077583197103195+0.316692813801149297542911 i$ & $6.7(-3)$ & $6.6(-4)$ & \\
\hline & 2 & $3.948542445561860453631143+0.316123570897181210401218 i$ & $2.5(-12)$ & $2.5(-13)$ & 4.497 \\
\hline & 3 & $3.948542445562045781056121+0.316123570897016377409433 i$ & $5.2(-50)$ & $5.1(-51)$ & 3.998 \\
\hline \multirow{4}{*}{$\mathrm{SH} 2$} & 0 & $3.9+0.25 i$ & $7.3(-1)$ & $8.2(-2)$ & \\
\hline & 1 & $3.949376481331022929997008+0.315510983543728513795726 i$ & $1.0(-2)$ & $1.0(-3)$ & \\
\hline & 2 & $3.948542445575461038975221+0.316123570894961364848795 i$ & $1.4(-10)$ & $1.4(-11)$ & 4.150 \\
\hline & 3 & $3.948542445562045781056121+0.316123570897016377409433 i$ & $4.0(-42)$ & $4.0(-43)$ & 4.000 \\
\hline \multirow{4}{*}{$Z S$} & 0 & $3.9+0.25 i$ & $7.3(-1)$ & $8.2(-2)$ & \\
\hline & 1 & $3.949276688095696783125591+0.315604814522904264479698 i$ & $9.1(-3)$ & $9.0(-4)$ & \\
\hline & 2 & $3.948542445569026701395342+0.316123570896386215539204 i$ & $7.1(-11)$ & $7.0(-12)$ & 4.136 \\
\hline & 3 & $3.948542445562045781056121+0.316123570897016377409433 i$ & $2.6(-43)$ & $2.6(-44)$ & 4.000 \\
\hline \multirow{4}{*}{ LS1 } & 0 & $3.9+0.25 i$ & $7.3(-1)$ & $8.2(-2)$ & \\
\hline & 1 & $3.949832856019659597809710+0.314572063857020040230532 i$ & $2.0(-2)$ & $2.0(-3)$ & \\
\hline & 2 & $3.948542445696559763673443+0.316123570640721245159885 i$ & $2.9(-9)$ & $2.9(-10)$ & 4.260 \\
\hline & 3 & $3.948542445562045781056121+0.316123570897016377409433 i$ & $1.2(-36)$ & $1.2(-37)$ & 4.000 \\
\hline \multirow{4}{*}{ LS2 } & 0 & $3.9+0.25 i$ & $7.3(-1)$ & $8.2(-2)$ & \\
\hline & 1 & $3.948773577799853969606873+0.315945221531233464343546 i$ & $3.0(-3)$ & $2.9(-4)$ & \\
\hline & 2 & $3.948542445562074039082972+0.316123570897003646179211 i$ & $3.1(-13)$ & $3.1(-14)$ & 4.073 \\
\hline & 3 & $3.948542445562045781056121+0.316123570897016377409433 i$ & $4.0(-53)$ & $3.9(-54)$ & 4.000 \\
\hline \multirow{4}{*}{$S A S$} & 0 & $3.9+0.25 i$ & $7.3(-1)$ & $8.2(-2)$ & \\
\hline & 1 & $3.948919691643135705506191+0.315869945878782590869753 i$ & $4.6(-3)$ & $4.5(-4)$ & \\
\hline & 2 & $3.948542445562318124465938+0.316123570896996715880628 i$ & $2.8(-12)$ & $2.7(-13)$ & 4.087 \\
\hline & 3 & $3.948542445562045781056121+0.316123570897016377409433 i$ & $3.6(-49)$ & $3.5(-50)$ & 4.000 \\
\hline \multirow{4}{*}{$(19)$} & 0 & $3.9+0.25 i$ & $7.3(-1)$ & $8.2(-2)$ & \\
\hline & 1 & $3.949067718859802129930896+0.315754069856917731302466 i$ & $6.5(-3)$ & $6.4(-4)$ & \\
\hline & 2 & $3.948542445563417859262609+0.316123570896854317633338$ & $1.4(-11)$ & $1.4(-12)$ & 4.115 \\
\hline & 3 & $3.948542445562045781056121+0.316123570897016377409433 i$ & $3.0(-46)$ & $3.0(-47)$ & 4.000 \\
\hline \multirow{4}{*}{$(20)$} & 0 & $3.9+0.25 i$ & $7.3(-1)$ & $8.2(-2)$ & \\
\hline & 1 & $3.948641405706844151768124+0.316024407295510931367185 i$ & $1.4(-3)$ & $1.4(-4)$ & \\
\hline & 2 & $3.948542445562046189031298+0.316123570897015499416852 i$ & $9.8(-15)$ & $9.7(-16)$ & 4.033 \\
\hline & 3 & $3.948542445562045781056121+0.316123570897016377409433 i$ & $2.2(-59)$ & $2.2(-60)$ & 4.000 \\
\hline
\end{tabular}

\section{Example 4. (Continuous stirred tank reactor (CSTR)):}

We assumed a problem of continuous stirred tank reactor (CSTR). We observed a reaction scheme that develops in the chemical reactor (see [20] for more information), which is defined as follows:

$$
\begin{aligned}
& W_{1}+T \rightarrow W_{2} \\
& W_{2}+T \rightarrow W_{3} \\
& W_{3}+T \rightarrow W_{4} \\
& W_{4}+T \rightarrow W_{5},
\end{aligned}
$$


where the components $T$ and $W_{1}$ are fed at the amount of $q-Q$ and $Q$, respectively, to the chemical reactor. The above model was studied in detail by Douglas [21] to find a good and simple system that can control feedback problem. Finally, he transferred the above model to the following mathematical expression:

$$
K_{H} \frac{2.98(x+2.25)}{(x+1.45)(x+4.35)(x+2.85)^{2}}=-1 \text {, }
$$

where $K_{H}$ denotes for the gaining proportional controller. The control system in Equation (29) is balanced with the values of $K_{H}$. If we assume $K_{H}=0$, we obtain the poles of the open-loop transferred function as the solutions of following uni-variate expression:

$$
\Theta_{4}(x)=x^{4}+11.50 x^{3}+47.49 x^{2}+83.06325 x+51.23266875=0
$$

given as: $x=-2.85,-1.45,-4.35$, and -2.85 . It is straightforward to say that we have one root $x=-2.85$, having known multiplicity 2. The computational results for Example 4 are displayed in Table 4.

\begin{tabular}{|c|c|c|c|c|c|}
\hline Methods & $l$ & $x_{l}$ & $\left|\Theta\left(x_{l}\right)\right|$ & $\left|x_{l+1}-x_{l}\right|$ & $\rho$ \\
\hline \multirow{4}{*}{ SH1 } & 0 & -0.28 & $5.2(-3)$ & $5.0(-2)$ & \\
\hline & 1 & -2.850000459297629356054243 & $4.4(-13)$ & $4.6(-7)$ & \\
\hline & 2 & -2.850000000000000000000000 & $5.4(-55)$ & $5.1(-28)$ & 44.161 \\
\hline & 3 & -2.850000000000000000000000 & $1.2(-222)$ & $7.5(-112)$ & 4.000 \\
\hline \multirow{4}{*}{ SH2 } & 0 & -0.28 & $5.2(-3)$ & $5.0(-2)$ & \\
\hline & 1 & -2.850000222747479938657484 & $1.0(-13)$ & $2.2(-7)$ & \\
\hline & 2 & -2.850000000000000000000000 & $7.2(-57)$ & $5.8(-29)$ & 4.033 \\
\hline & 3 & -2.850000000000000000000000 & $1.6(-229)$ & $2.8(-115)$ & 4.000 \\
\hline \multirow{4}{*}{$Z S$} & 0 & -0.28 & $5.2(-3)$ & $5.0(-2)$ & \\
\hline & 1 & -2.850000145480769072643415 & $4.4(-14)$ & $1.5(-7)$ & \\
\hline & 2 & -2.850000000000000000000000 & $5.5(-59)$ & $5.1(-30)$ & 4.056 \\
\hline & 3 & -2.850000000000000000000000 & $1.2(-238)$ & $7.7(-120)$ & 4.000 \\
\hline \multirow{4}{*}{$L S 1$} & 0 & -0.28 & $5.2(-3)$ & $5.0(-2)$ & \\
\hline & 1 & -2.850000145138892814208824 & $4.4(-14)$ & $1.5(-7)$ & \\
\hline & 2 & -2.850000000000000000000000 & $5.4(-59)$ & $5.0(-30)$ & 4.056 \\
\hline & 3 & -2.850000000000000000000000 & $1.1(-238)$ & $7.4(-120)$ & 4.000 \\
\hline \multirow{4}{*}{$L S 2$} & 0 & -0.28 & $5.2(-3)$ & $5.0(-2)$ & \\
\hline & 1 & -2.850000145138892814208824 & $4.4(-14)$ & $1.5(-7)$ & \\
\hline & 2 & -2.850000000000000000000000 & $5.4(-59)$ & $5.0(-30)$ & 4.056 \\
\hline & 3 & -2.850000000000000000000000 & $1.1(-238)$ & $7.4(-120)$ & 4.000 \\
\hline \multirow{4}{*}{$S A S$} & 0 & -0.28 & $5.2(-3)$ & $5.0(-2)$ & \\
\hline & 1 & -2.850000145309626720076681 & $4.4(-14)$ & $1.5(-7)$ & \\
\hline & 2 & -2.850000000000000000000000 & $5.4(-59)$ & $5.1(-30)$ & 4.056 \\
\hline & 3 & -2.850000000000000000000000 & $1.2(-238)$ & $7.5(-120)$ & 4.000 \\
\hline \multirow{4}{*}{ (19) } & 0 & -0.28 & $5.2(-3)$ & $5.0(-2)$ & \\
\hline & 1 & -2.850000145138892814208824 & $4.4(-14)$ & $1.5(-7)$ & \\
\hline & 2 & -2.850000000000000000000000 & $5.4(-59)$ & $5.0(-30)$ & 4.056 \\
\hline & 3 & -2.850000000000000000000000 & $1.1(-238)$ & $7.4(-120)$ & 4.000 \\
\hline \multirow{4}{*}{$(20)$} & 0 & -0.28 & $5.2(-3)$ & $5.0(-2)$ & \\
\hline & 1 & -2.850000145025115304550159 & $4.4(-14)$ & $1.5(-7)$ & \\
\hline & 2 & -2.850000000000000000000000 & $5.3(-59)$ & $5.0(-30)$ & 4.056 \\
\hline & 3 & -2.850000000000000000000000 & $1.1(-238)$ & $7.3(-120)$ & 4.000 \\
\hline
\end{tabular}

Table 4. Convergence study of distinct iterative functions on $\Theta_{4}(x)$. 
Example 5. We considered an academic problem, which is given as follows:

$$
\Theta_{5}(x)=-\sum_{j=0}^{p} \frac{x^{j}}{j !}+\exp (x), \quad p=20 .
$$

Equation (31) has a zero $x=0$ of multiplicity 20.

Table 5. Convergence study of distinct iterative functions on $\Theta_{5}(x)$.

\begin{tabular}{|c|c|c|c|c|c|}
\hline Methods & $l$ & $x_{l}$ & $\left|\Theta\left(x_{l}\right)\right|$ & $\left|x_{l+1}-x_{l}\right|$ & $\rho$ \\
\hline \multirow{4}{*}{ SH1 } & 0 & 0.5 & $4.0(-25)$ & $5.0(-1)$ & \\
\hline & 1 & -0.0002718978528821532806208018 & $2.0(-90)$ & $2.7(-4)$ & \\
\hline & 2 & $1.694191703400450566540429(-20)$ & $1.6(-414)$ & $1.7(-20)$ & 4.964 \\
\hline & 3 & $5.950691975131256213603499(-86)$ & $1.3(-1723)$ & $6.0(-86)$ & 4.039 \\
\hline \multirow{4}{*}{$\mathrm{SH} 2$} & 0 & 0.5 & $4.0(-25)$ & $5.0(-1)$ & \\
\hline & 1 & -0.00002193761299309735772091143 & $2.7(-112)$ & $2.2(-5)$ & \\
\hline & 2 & $-8.159736153943269589123114(-23)$ & $7.0(-461)$ & $8.2(-23)$ & 4.000 \\
\hline & 3 & $-1.561791403734642244424094(-92)$ & $3.1(-1855)$ & $1.6(-92)$ & 4.000 \\
\hline \multirow{4}{*}{$Z S$} & 0 & 0.5 & $4.0(-25)$ & $5.0(-1)$ & \\
\hline & 1 & $4.704138277920796230225854(-8)$ & $1.2(-165)$ & $4.7(-8)$ & \\
\hline & 2 & $3.542974646434738595665076(-36)$ & $4.0(-728)$ & $3.5(-36)$ & 4.002 \\
\hline & 3 & $1.140039512445237589479550(-148)$ & $5.7(-2978)$ & $1.1(-148)$ & 4.000 \\
\hline \multirow{4}{*}{$L S 1$} & 0 & 0.5 & $4.0(-25)$ & $5.0(-1)$ & \\
\hline & 1 & $4.703388329419000312031088(-8)$ & $1.2(-165)$ & $4.7(-8)$ & \\
\hline & 2 & $3.540164953595947998723919(-36)$ & $3.9(-728)$ & $3.5(-36)$ & 4.002 \\
\hline & 3 & $1.136250641816172375721875(-148)$ & $5.3(-2978)$ & $1.1(-148)$ & 4.000 \\
\hline \multirow{4}{*}{ LS2 } & 0 & 0.5 & $4.0(-25)$ & $5.0(-1)$ & \\
\hline & 1 & $4.703320825841908042922536(-8)$ & $1.2(-165)$ & $4.7(-8)$ & \\
\hline & 2 & $3.539912143976027683786300(-36)$ & $3.9(-728)$ & $3.5(-36)$ & 4.002 \\
\hline & 3 & $1.135910200587531412808986(-148)$ & $5.3(-2978)$ & $1.1(-148)$ & 4.000 \\
\hline \multirow{4}{*}{$S A S$} & 0 & 0.5 & $4.0(-25)$ & $5.0(-1)$ & \\
\hline & 1 & $4.704063972314087734965678(-8)$ & $1.2(-165)$ & $4.7(-8)$ & \\
\hline & 2 & $3.542696174326264352804257(-36)$ & $4.0(-728)$ & $3.5(-36)$ & 4.002 \\
\hline & 3 & $1.139663562372889176842286(-148)$ & $5.6(-2978)$ & $1.1(-148)$ & 4.000 \\
\hline \multirow{4}{*}{ (19) } & 0 & 0.5 & $4.0(-25)$ & $5.0(-1)$ & \\
\hline & 1 & $4.703284202716149126456111(-8)$ & $1.2(-165)$ & $4.7(-8)$ & \\
\hline & 2 & $3.539774991786613338951462(-36)$ & $3.9(-728)$ & $3.5(-36)$ & 4.002 \\
\hline & 3 & $1.135725539761171832682598(-148)$ & $5.2(-2978)$ & $1.1(-148)$ & 4.000 \\
\hline \multirow{4}{*}{$(20)$} & 0 & 0.5 & $4.0(-25)$ & $5.0(-1)$ & \\
\hline & 1 & $4.703247172631055037496472(-8)$ & $1.2(-165)$ & $4.7(-8)$ & \\
\hline & 2 & $3.539636320143384844486839(-36)$ & $3.9(-728)$ & $3.5(-36)$ & 4.002 \\
\hline & 3 & $1.135538856470940547850597(-148)$ & $5.2(-2978)$ & $1.1(-148)$ & 4.000 \\
\hline
\end{tabular}

Example 6. We picked another academic problem from Zeng [22]:

$$
\Theta_{6}(x)=(x-2)^{15}(x-4)^{5}(x-3)^{10}(x-1)^{20} .
$$

In the above function, we have four multiple zeros $x=1,2,3$ and 4 and multiplicity of the corresponding zero is 20, 15, 10 and 5, respectively. All multiple zero are quite close to each other. We chose $x=1$ multiple zero of multiplicity 20 for the computational point of view and the results are depicted in Table 6 . 
Table 6. Convergence study of distinct iterative functions on $\Theta_{6}(x)$.

\begin{tabular}{|c|c|c|c|c|c|}
\hline Methods & $l$ & $x_{l}$ & $\left|\Theta\left(x_{l}\right)\right|$ & $\left|x_{l+1}-x_{l}\right|$ & $\rho$ \\
\hline \multirow{4}{*}{ SH1 } & 0 & 1.1 & $2.6(-16)$ & $1.0(-1)$ & \\
\hline & 1 & 0.9989451141946186671826623 & $7.4(-55)$ & $1.1(-3)$ & \\
\hline & 2 & 0.9999999999940157811870657 & $8.6(-220)$ & $6.0(-12)$ & 4.162 \\
\hline & 3 & 1.000000000000000000000000 & $2.0(-879)$ & $6.2(-45)$ & 4.000 \\
\hline \multirow{4}{*}{$\mathrm{SH} 2$} & 0 & 1.1 & $2.6(-16)$ & $1.0(-1)$ & \\
\hline & 1 & 0.9989452078176884754918726 & $7.4(-55)$ & $1.1(-3)$ & \\
\hline & 2 & 0.9999999999940174546383277 & $8.6(-220)$ & $6.0(-12)$ & 4.162 \\
\hline & 3 & 1.000000000000000000000000 & $2.0(-879)$ & $6.2(-45)$ & 4.000 \\
\hline \multirow{4}{*}{$Z S$} & 0 & 1.1 & $2.6(-16)$ & $1.0(-1)$ & \\
\hline & 1 & 0.9989316527404047970937760 & $9.6(-55)$ & $1.1(-3)$ & \\
\hline & 2 & 0.9999999999935564098598278 & $3.8(-219)$ & $6.4(-12)$ & 4.160 \\
\hline & 3 & 1.000000000000000000000000 & $1.2(-876)$ & $8.6(-45)$ & 4.000 \\
\hline \multirow{4}{*}{ LS1 } & 0 & 1.1 & $2.6(-16)$ & $1.0(-1)$ & \\
\hline & 1 & 0.9989328999398545622800000 & $9.3(-55)$ & $1.1(-3)$ & \\
\hline & 2 & 0.9999999999936000861495460 & $3.3(-219)$ & $6.4(-12)$ & 4.160 \\
\hline & 3 & 1.000000000000000000000000 & $6.6(-877)$ & $8.3(-45)$ & 4.000 \\
\hline \multirow{4}{*}{ LS2 } & 0 & 1.1 & $2.6(-16)$ & $1.0(-1)$ & \\
\hline & 1 & 0.9989330109658531082468530 & $9.3(-55)$ & $1.1(-3)$ & \\
\hline & 2 & 0.9999999999936039765023947 & $3.3(-219)$ & $6.4(-12)$ & 4.160 \\
\hline & 3 & 1.000000000000000000000000 & $6.3(-877)$ & $8.3(-45)$ & 4.000 \\
\hline \multirow{4}{*}{$S A S$} & 0 & 1.1 & $2.6(-16)$ & $1.0(-1)$ & \\
\hline & 1 & 0.9989317774496343892296356 & $9.5(-55)$ & $1.1(-3)$ & \\
\hline & 2 & 0.9999999999935607750796670 & $3.7(-219)$ & $6.4(-12)$ & 4.160 \\
\hline & 3 & 1.000000000000000000000000 & $1.1(-876)$ & $8.6(-45)$ & 4.000 \\
\hline \multirow{4}{*}{ (19) } & 0 & 1.1 & $2.6(-16)$ & $1.0(-1)$ & \\
\hline & 1 & 0.9989330711806011592330876 & $9.3(-55)$ & $1.1(-3)$ & \\
\hline & 2 & 0.9999999999936060858850038 & $3.2(-219)$ & $6.4(-12)$ & 4.160 \\
\hline & 3 & 1.000000000000000000000000 & $6.1(-877)$ & $8.3(-45)$ & 4.000 \\
\hline \multirow{4}{*}{$(20)$} & 0 & 1.1 & $2.6(-16)$ & $1.0(-1)$ & \\
\hline & 1 & 0.9989331320096201097185023 & $9.3(-55)$ & $1.1(-3)$ & \\
\hline & 2 & 0.9999999999936082168412861 & $3.2(-219)$ & $6.4(-12)$ & 4.160 \\
\hline & 3 & 1.000000000000000000000000 & $5.9(-877)$ & $8.3(-45)$ & 4.000 \\
\hline
\end{tabular}

Remark 1. The main benefit of this paper is that we suggest a new family of fourth-order Schröder method having optimal convergence.

Remark 2. Finding a weight function with some pre-defined conditions is not an easy task. With the help of free disposable parameters, we can construct several new iterative schemes. Additionally, we also present some existing methods, i.e., those of Zhou et al. [9] and Li et al. [7], as special cases of our scheme (see Equations (20) and (23), respectively).

Remark 3. It is important to note that Equation (4), consumes only three new functional values $\Theta\left(x_{l}\right), \Theta^{\prime}\left(x_{l}\right)$ and $\Theta^{\prime}\left(y_{l}\right)$, at each iteration. Thus, Theorem 1 shows that new scheme in Equation (4) has optimal fourth-order convergence, as predicted by the Kung-Traub conjecture [4].

Remark 4. Our methods also work for simple and complex roots of nonlinear equations.

\section{Concluding Remarks}

We construct a new optimal family of Schröder's method for computing the multiple zeros of uni-variate nonlinear functions. The presented scheme in Equation (9) adopted only one value of $\Theta$ and two of derivative $\Theta^{\prime}$ at each step. Our methods do not use the values of second or high-order 
derivatives. The principle benefit of our family is that there is no need of weight functions to further construct new optimal fourth-order methods. We also show that we can easily obtain several new and some existing classical techniques by adjusting one of the parameters from $\alpha_{1}$ and/or $\alpha_{2}$. We conclude from numerical experimentation that our methods have at least equal performance as compared to ZS, LS1, LS2, SAS and also demonstrate better behavior than SH1 and SH2 in Examples 1, 2, and 4-6. In Example 3, our method in Equation (20) illustrates the better performance as compared to all other considered methods and the results can be seen in Table 3.

Funding: This research received no external funding.

Author Contributions: R.B.: Conceptualization; Methodology; Validation; Writing-Original Draft Preparation; Writing-Review \& Editing. A.J.A.: Validation; Review \& Editing. B.A.P., W.M.A.-H., M.S. and M.F.: Review \& Editing.

Conflicts of Interest: The authors declare no conflict of interest.

\section{References}

1. Behl, R.; Salimi, M.; Ferrara, M.; Sharifi, S.; Samaher, K.A. Some real life applications of a newly constructed derivative free iterative scheme. Symmetry 2019, 11, 239. [CrossRef]

2. Salimi, M.; Nik Long, N.M.A.; Sharifi, S.; Pansera, B.A. A multi-point iterative method for solving nonlinear equations with optimal order of convergence. Jpn. J. Ind. Appl. Math. 2018, 35, 497-509. [CrossRef]

3. Sharifi, S.; Salimi, M.; Siegmund, S.; Lotfi, T. A new class of optimal four-point methods with convergence order 16 for solving nonlinear equations. Math. Comput. Simul. 2016, 119, 69-90. [CrossRef]

4. Kung, H.T.; Traub, J.F. Optimal order of one-point and multipoint iteration. J. Assoc. Comput. Mach. 1974, 21, 643-651. [CrossRef]

5. Rall, L.B. Convergence of Newton's process to multiple solutions. Numer. Math. 1966, 9, 23-37. [CrossRef]

6. Li, S.G.; Cheng, L.Z.; Neeta, B. Some fourth-order nonlinear solvers with closed formulae for multiple roots. Comput. Math. Appl. 2010, 59, 126-135. [CrossRef]

7. Li, S.; Liao, X.; Cheng, L. A new fourth-order iterative method for finding multiple roots of nonlinear equations. Appl. Math. Comput. 2009, 215, 1288-1292.

8. Sharma, J.R.; Sharma, R. Modified Jarratt method for computing multiple roots. Appl. Math. Comput. 2010, 217, 878-881. [CrossRef]

9. Zhou, X.; Chen, X.; Song, Y. Constructing higher-order methods for obtaining the muliplte roots of nonlinear equations. J. Comput. Appl. Math. 2011, 235, 4199-4206. [CrossRef]

10. Kumar, S.; Kanwar, V.; Singh, S. On some modified families of multipoint iterative methods for multiple roots of nonlinear equations. Appl. Math. Comput. 2012, 218, 7382-7394. [CrossRef]

11. Neta, B.; Jhonson, A.N. High order nonlinear solver for multiple roots. Comput. Math. Appl. 2008, 55, 2012-2017. [CrossRef]

12. Khattri, S.K.; Abbasbandy, S. Optimal fourth order family of iterative methods. Mat. Vesn. 2011, 63, 67-72.

13. Schröder, E. Ueber unendlich viele Algorithmen zur Auflösung der Gleichungen. Math. Ann. 1870, 2, 317-365. [CrossRef]

14. Traub, J.F. Iterative Methods for the Solution of Equations; Prentice-Hall: Englewood Cliffs, NJ, USA, 1964.

15. Sharifi, M.; Babajee, D.K.R.; Soleymani, F. Finding the solution of nonlinear equations by a class of optimal methods. Comput. Math. Appl. 2012, 63, 764-774. [CrossRef]

16. Weerakoon, S.; Fernando, T.G.I. A variant of Newton's method with accelerated third-order convergence. Appl. Math. Lett. 2000, 13, 87-93. [CrossRef]

17. Ezquerro, J.A.; Hernández, M.A. New iterations of R-order four with reduced computational cost. BIT Numer. Math. 2009, 49, 325-342. [CrossRef]

18. Shacham, M. An improved memory method for the solution of a nonlinear equation. Chem. Eng. Sci. 1989, 44,1495-1501. [CrossRef]

19. Balaji, G.V.; Seader, J.D. Application of interval Newton's method to chemical engineering problems. Reliab. Comput. 1995, 1, 215-223. [CrossRef]

20. Constantinides, A.; Mostoufi, N. Numerical Methods for Chemical Engineers with MATLAB Applications; Prentice Hall PTR: Upper Saddle River, NJ, USA, 1999. 
21. Douglas, J.M. Process Dynamics and Control; Prentice Hall: Englewood Cliffs, NJ, USA, 1972; Volume 2.

22. Zeng, Z. Computing multiple roots of inexact polynomials. Math. Comput. 2004, 74, 869-903. [CrossRef]

(C) 2019 by the authors. Licensee MDPI, Basel, Switzerland. This article is an open access article distributed under the terms and conditions of the Creative Commons Attribution (CC BY) license (http:/ / creativecommons.org/licenses/by/4.0/). 